earlier than ordinary fluid treatment, especially oxygen encephalography seems to be much better than air one.

\title{
157. Traumatic Aneurysm located at the Depth of Extracranial Tissues
}

\author{
Morio Matsunaga, Tetsuaki Teraura and Syun Yamasaki \\ Department of Neurosurgery, Kurashiki Central Hospital
}

It has been noticed within last decade or so that the various type of vascular deseases besides the carotid cavernous fistula gives rise to posttraumatically. Traumatic aneurysm is one of them. Few reports have been concerned with the true aneurysm where its wall was composed of proper components of vessel organ. Most of the cases reported up to now have, however, been of the false aneurysm situated in the cranial cavity.

We have found 3 cases of traumatic aneurysm (including one un-operated case) located at the depth of extracranial soft tissues. Only one patient was female. The age ranged 23 to 45 . In one male patient, an aneurysm of ca $10 \mathrm{~mm}$. in diameter was $2 \mathrm{~cm}$. apart from the other one of ca $5 \mathrm{~mm}$. All 3 aneurysms excised were fed from the superficial temporal artery. Every EEGraphic record taken from the patients showed principally neither asymmetry nor focal abnormalities, suggesting any brain contusion. One exceptional patient (un-operated) had suffered from the otitis media long before the trauma. In one case, definite focal abnormality was revealed successively by means of the impedance prethysmography.

Covering skin was protruding and pulsating in 3 operated aneurysms. Fresh scar resected from sutures was also seen within $1 \mathrm{~cm}$. or so in this group. In the remaining case, on the contrary, the scalp seemed intact. The feeding artery was easily photographied in all 4 aneurysms (in angiograms). All the operated ones were saccular in type, and recognized even in the venous phase $(5$ second from the termination of the injection). Differing from the aneurysm fed from the middle meningeal artery, the mass of the contrast media was situated outside of the outer table in the A-P view. Local anesthesia was usually used at the minor surgery room.

The common HE staining, the elastic fibre staining (Weigert) and the Masson's staining were appointed. In a short conclusion in our study, all the excised aneurysms were of the false. Otherwise, neither the media nor the intima was certified as the wall structures. The internal elastic lamina was missing. 